University of Nebraska - Lincoln

DigitalCommons@University of Nebraska - Lincoln

Faculty Publications: Department of Entomology

2021

Toxicological analysis of stilbenes against the fall armyworm, Spodoptera frugiperda

Sarah E. McComic

Leslie C. Rault

Troy D. Anderson

Daniel R. Swale

Follow this and additional works at: https://digitalcommons.unl.edu/entomologyfacpub

Part of the Biochemistry, Biophysics, and Structural Biology Commons, and the Entomology Commons

This Article is brought to you for free and open access by the Entomology, Department of at

DigitalCommons@University of Nebraska - Lincoln. It has been accepted for inclusion in Faculty Publications:

Department of Entomology by an authorized administrator of DigitalCommons@University of Nebraska - Lincoln. 


\title{
Toxicological analysis of stilbenes against the fall armyworm, Spodoptera frugiperda
}

\author{
Sarah E. McComic, ${ }^{1}$ Leslie C. Rault, ${ }^{2}$ \\ Troy D. Anderson, ${ }^{2}$ and Daniel R. Swale ${ }^{1}$
}

\begin{abstract}
1 Louisiana State University AgCenter, Department of Entomology, Baton Rouge, LA 70803

2 University of Nebraska, Department of Entomology, 103 Entomology Hall, Lincoln, NE 68583

Corresponding author - D.R. Swale, 404 Life Sciences Building, LSU, Department of Entomology, Baton Rouge, LA 70803, USA Email dswale@agcenter.lsu.edu
\end{abstract}

\begin{abstract}
The fall armyworm (FAW), Spodoptera frugiperda, is a global pest of multiple economically important row crops and the development of resistance to commercially available insecticidal classes has inhibited FAW control. Thus, there is a need to identify chemical scaffolds that can provide inspiration for the development of novel insecticides for FAW management. This study aimed to assess the sensitivity of central neurons and susceptibility of FAW to chloride channel modulators to establish a platform for repurposing existing insecticides or designing new chemicals capable of controlling FAW. Potency of select chloride channel modulators were initially studied against FAW central neuron firing rate and rank order of potency was determined to be fipronil $>$ lindane $>Z$-stilbene $>$ DIDS $>$ GABA $>E$-stilbene. Toxicity bioassays identified fipronil and lindane as the two most toxic modulators studied with topical $\mathrm{LD}_{50}$ 's of 41 and $75 \mathrm{ng} / \mathrm{mg}$ of caterpillar, respectively. Interestingly, $Z$-stilbene was toxic at $300 \mathrm{ng} / \mathrm{mg}$ of caterpillar, but no toxicity was observed with DIDS or $E$-stilbene. The significant shift in potency between stilbene isomers
\end{abstract}

Published in Pesticide Biochemistry and Physiology (2021)

doi:10.1016/j.pestbp.2021.104965

Copyright (C) 2021. Published by Elsevier. Used by permission.

Submitted 9 June 2021; revised 17 August 2021; accepted 14 September 2021. 
indicates structure-activity relationships between stilbene chemistry and the binding site in FAW may exist. The data presented in this study defines the potency of select chloride channel modulators to FAW neural activity and survivorship to establish a platform for development of novel chemical agents to control FAW populations. Although stilbenes may hold promise for insecticide development, the low toxicity of the scaffolds tested in this study dampen enthusiasm for their development into FAW specific insecticides.

Keywords: Insecticide resistance, Insecticide development, Neurophysiology, Fall armyworm, Chloride channel, DIDS, Stilbene

\section{Introduction}

The fall armyworm (FAW), Spodoptera frugiperda (Lepidoptera: Noctuidae), is recognized as one of the most damaging agricultural pests of row crops with economic losses estimated to be $\$ 6$ billion dollars per year, which primarily stems from FAW infestation of maize crop (Yu et al., 2003). The FAW range has expanded from the Americas to nearly 100 countries (Goergen et al., 2016; Sharanabasappa et al., 2018; Wu et al., 2019; Cairns et al., 2013), which has threatened the food security of millions of people due to the ability of FAW to cause $100 \%$ loss of maize and rice crop if left uncontrolled. FAW control programs rely on a combination of synthetic insecticides and $B$. thuringiensis $(B t)$ expressing plants to maintain pest populations below the economic damage threshold (Brookes and Barfoot, 2016; Blanco et al., 2016; Gutierrez- Moreno et al., 2019). An increase in Cry1F resistance alleles within FAW populations throughout the Americas (Blanco et al., 2016; Santos- Amaya et al., 2017) has prompted farmers to significantly increase the frequency of pyrethroid and organophosphate applications to maintain low FAW levels (Houngbo et al., 2020; Kansiime et al., 2019). Unsurprisingly, the increased frequency of application drove the evolution of resistance to multiple insecticidal classes, including pyrethroids, organophosphates, carbamates, benzoylureas, spinosyns, and diamides (Rios-Diez and Saldamando-Benjumea, 2011; Okuma et al., 2018; Jia et al., 2009; Carvalho et al., 2013; Boaventura et al., 2020). The widespread resistance to multiple classes of insecticides emphasizes the need to study additional target sites (e.g., ligand-gated chloride $\left(\mathrm{Cl}^{-}\right)$channels (LGCC), voltage-dependent chloride channels (VDCC)) that can provide selective toxicity to control FAW populations. 
Membrane bound proteins containing chloride ion channels are longstanding targets for insecticides, acaricides, and anthelmintics (Bloomquist, 2003). For example, the GABA- and glutamate-gated chloride channel complexes contains drug-binding sites for multiple chemical classes, such as cyclodienes, phenylpyrazoles, macrocyclic lactones, and meta-diamides, which are all established agrochemicals for management of lepidopteran pests (Casida and Durkin, 2015; Nakao et al., 2013; Ozoe et al., 2010; Asahi et al., 2015). The firstgeneration GABAergic insecticides consisted of multiple commercial compounds classified into three primary chemical categories, the polychlorocycloalkanes (e.g., lindane and toxaphene), cyclodienes (e.g., dieldrin and endosulfan), and phenylpyrazoles (e.g., fipronil) (Casida and Durkin, 2015). However, the target-site mutations in $r d l$, the gene encoding GABA-R, has significantly reduced the utility of conventional GABAergic insecticides. The reduced efficacy of first-generation GABAergic insecticides led to the development of second-generation GABAergic insecticides, such as meta-diamides, that are toxic to a wide variety of arthropod pests, including lepidopterans (Casida and Durkin, 2015; Jiang et al., 2017; Miglianico et al., 2018; Nakao and Banba, 2016). The development of these molecules has broadened the diversity of chemistries targeting ligand-gated chloride $\left(\mathrm{Cl}^{-}\right)$channels (LGCC) due to the lack of cross-resistance resulting from their action at a different, high affinity site when compared to first generation molecules (Asahi et al., 2015; Ozoe et al., 2013).

In addition to LGCC, the critical VDCC provide to neurotransmission (Fahlke, 2001), combined with the fact some GABA antagonists interact with VDCC (Bloomquist, 1993), has driven suggestions that VDCC are promising targets for development of selective insecticides (Bloomquist, 2003; Bloomquist, 1996) and miticides (Vu et al., 2020). Unfortunately, chemical leads to target FAW VDCC are not well described in the literature and this gap in knowledge has slowed the development of VDCC as a target site. The synthetic stilbene derivative 4,4'-Diisothiocyano-2,2'- stilbenedisulfonic acid (DIDS) is an established blocker of VDCC (Abalis et al., 1986) that elicits paralytic activity of invertebrates through blockage of chloride ion flux (Bloomquist, 2003; Hu et al., 1999). DIDS and other anion-transport blockers have been considered promising candidate molecules with unique modes of action for the management of the honey bee ectoparasite Varroa 
destructor ( $\mathrm{Vu}$ et al., 2020) and the crop pest $O$. nubilalis (Boina and Bloomquist, 2009), suggesting that stilbene analogs may provide inspiration for the development of VDCC-directed molecules to manage FAW.

The threat to global food security and the dwindling efficacy of commercialized insecticides to control FAW infestations underscores the need to identify chemical scaffolds that can provide inspiration for the development of novel insecticides for the management of lepidopteran pests. Thus, the goal of this study was to employ physiological and toxicological approaches to assess the sensitivity of the central nervous system (CNS) and susceptibility of FAW to LGCC and VDCC modulators that may provide insight into directions for novel insecticide design for this lepidopteran pest.

\section{Methods}

\subsection{Compounds and compound synthesis}

DIDS ( \pm ), fipronil, lindane, cis ( $Z$ )- and trans $(E)$-stilbene, $\alpha$-phenylcinnamonitrile, $E$ - $\alpha$-methylstilbene, 4,4'-Bis(2-benzoxazolyl)stilbene, 2,4-dinitro-3',4'-(methylenedioxy)stilbene, and gammaaminobutyric acid (GABA) were all purchased from Sigma-Aldrich Chemical Co. (St. Louis, MO, USA). All compounds were > 95\% purity. Structures of stilbenes used in this study are shown in Figure 1. The solvents dimethyl sulfoxide (DMSO) and absolute ethanol were purchased from Sigma-Aldrich Chemical Co. A molecular sieve OP type 3 $\AA$ was purchased from Sigma-Aldrich and used to prevent water absorption within the DMSO stock.

\subsection{Spodoptera frugiperda stock and rearing conditions}

The laboratory colony of FAW used in this study, referred to as LSULab-1 (McComic et al., 2020), was initially established in 2005 from cotton fields at the Macon Ridge Research Station in Winnsboro, Louisiana. LSU-Lab-1 was genetically confirmed as being the corn-strain (McComic et al., 2020) and was maintained as previously described (McComic et al., 2020; Gordy et al., 2015). Caterpillars were reared on 


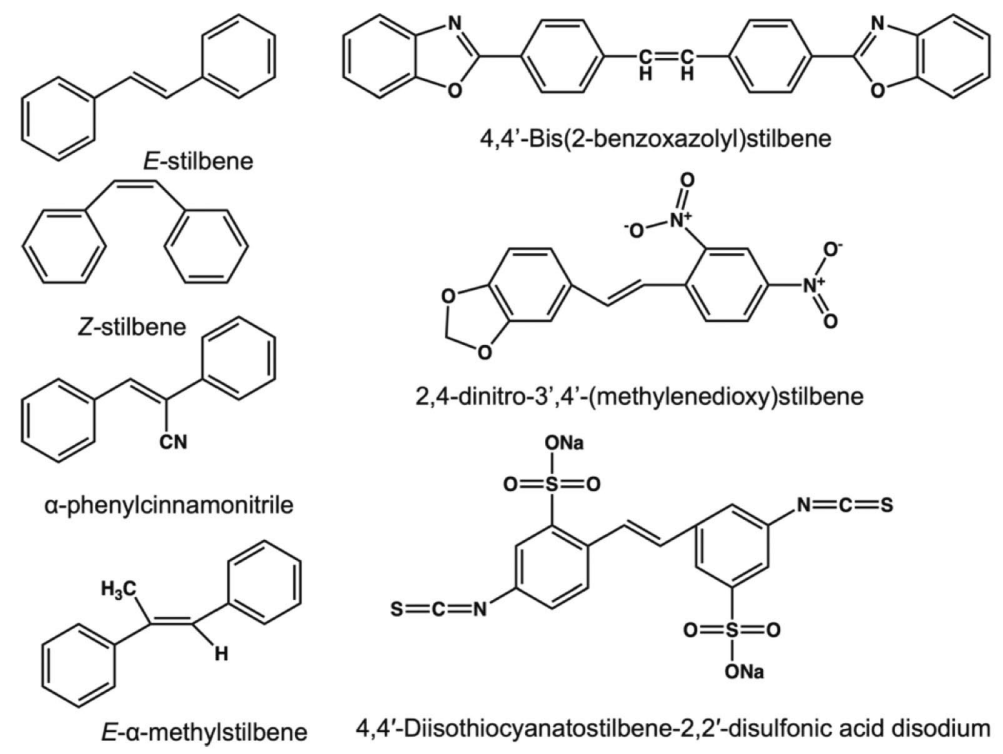

Figure 1. Molecular structures of stilbenes.

artificial diet (Stonefly Heliothis Diet, Ward's Natural Science, Rochester, NY, USA) in 30-mL plastic cups. Eggs were deposited on the cheesecloth and 20-30 neonates were placed in eight-cell trays (BioServ, Frenchtown, NJ, USA) as they emerged. Caterpillars for experiments were kept on diet for approximately 7-8 d until they exhibited signs of molting and were synchronized at head capsule slippage.

\subsection{In silico analyses of FAW chloride channel complexes}

An analysis of chloride channel complexes was conducted using a FAW partially annotated genome available on NCBI (Xiao et al., 2020). The transcripts were identified from the genome to extract mRNA sequences annotated as chloride channels. A similar process was conducted for chloride channel complexes of $D$. melanogaster (reference genome available on NCBI: Dmel_GCF_ooooo1215.4_Release_6 plus ISO1 MT) and H. sapiens (Human_GCF_ooooo1405.39_GRCh38.p13, also available on NCBI). The sequences with clearly annotated transcripts and representative transcript variants for each major annotation of $D$. melanogaster and $H$. sapiens chloride channels were selected for a multiple sequence alignment using Clustal Omega (Sievers et al., 2011). The alignment was then converted into a neighbor-joining 
tree of phylogenetic relationships based on sequence similarities. The tree was visualized and modified with FigTree v1.4.4 ( $\mathrm{http}$ ://tree.bio. ed.ac.uk/software/figtree/ , accessed 07/13/2020.)

\subsection{Neurophysiological assays}

Extracellular recordings of spontaneous neuronal activity from the FAW CNS followed our previous publications (McComic et al., 2020). The central nervous system was dissected from third-instar FAW, manually transected between ganglia to disrupt the blood brain barrier, and bathed in $200 \mu \mathrm{L}$ physiological saline (Salgado et al., 1998) containing: $140 \mathrm{mM} \mathrm{NaCl}, 5 \mathrm{mM} \mathrm{KCl}, 4 \mathrm{mM} \mathrm{CaCl}_{2}, 5 \mathrm{mM}$ HEPES, $28 \mathrm{mM}$ Dglucose, pH: 7.4. Peripheral nerve trunks from the thoracic or abdominal ganglia were drawn into the suction electrodes and electrical descending nerve activity was amplified by an AC/DC amplifier (Model 1700, Systems, Inc., Carlsborg, WA, USA), subjected to window amplitude discrimination, and converted on-line into a rate plot. Noise (6o Hz) was eliminated using Hum Bug (A-M Systems, Sequim, WA, USA). The firing rate was expressed in Hertz (Hz) using LabChart7 Pro (ADInstruments, Colorado Springs, CO, USA). Neural firing rates were monitored and measured following our previously described methods (McComic et al., 2020). For construction of concentration response curves, firing frequencies were measured for 3-5 min for each concentration prior to the addition of the next drug concentration. Individual concentrations were performed on individual preparations to ensure the effect to CNS firing was not due to sequential application of increasing drug concentrations. Mean spike discharge frequencies over the entire 3-5-min recording period for each concentration were used to construct concentration-response curves to determine $\mathrm{EC}_{50}$ (i.e., concentration that elicits $50 \%$ excitation) and $\mathrm{IC}_{50}$ (i.e., concentration that elicits $50 \%$ inhibition) values that were calculated by nonlinear regression (variable slope) using GraphPad Prism ${ }^{\mathrm{TM}} 9$ (GraphPad Software, San Diego, CA, USA). Each drug concentration was replicated $5^{-10}$ times.

\subsection{Insecticide toxicity bioassays}

The laboratory susceptibility of third-instar caterpillars to $\mathrm{Cl}^{-}$channel modulators was determined by ingestion, microinjection, and topical 
bioassays. For ingested toxicity and developmental bioassays, chemicals were incorporated in the diet at $100 \mu \mathrm{g} / \mathrm{mL}$ of media and neonates were placed individually on a treated diet cup. For topical bioassays, caterpillars were treated with $1 \mu \mathrm{L}$ of $95 \%$ ethanol (control) or ethanol containing the insecticide applied to the thoracic dorsum using a hand-held pipette (Gilson, Middleton, WI, USA). For microinjections, $69 \mathrm{~nL}$ of DMSO control or DMSO containing the insecticide was injected between the first and second abdominal segments via a World Precision Instruments (WPI) Nanoliter 2010 injector operated by a WPI SMARTouch controller. Treated caterpillars were held at 25 ${ }^{\circ} \mathrm{C}$ with a 14:10 h (light:dark) photoperiod and mortality was assessed at 48-h post treatment. Mortality was defined as the inability for coordinated movement within $10 \mathrm{~s}$ after prodding with a needle. The dose required to kill $50 \%$ of the population (lethal dose, $\mathrm{LD}_{50}$ ) was calculated using GraphPad Prism (GraphPad Software, San Diego, CA) and was constructed using 6-9 concentrations that ranged from $0 \%$ to $100 \%$ mortality. Each concentration consisted of three replicates of 10 caterpillars/replicate. A total of three $\mathrm{LD}_{50}$ values were obtained from separate cohorts, and the mean $\mathrm{LD}_{50}$ value was used for the generation of the resistance ratio (field colony $\mathrm{LD}_{50}$ /laboratory colony $\mathrm{LD}_{50}$ ). For all toxicity bioassays, control mortality never exceeded $10 \%$ and Abbots formula (Abbot, 1925) was used to correct for control mortality.

Microinjection of stilbenes were limited due to poor solubility of most molecules studied and, thus, the percent toxicity reported was based on solubility limits for each compound. The doses studied are: $300 \mathrm{ng} / \mathrm{mg}$ for $Z$-stilbene, $100 \mathrm{ng} / \mathrm{mg}$ for $E$-stilbene, $400 \mathrm{ng} / \mathrm{mg}$ for $\alpha$-phenylcinnamonitrile, $16 \mathrm{ng} / \mathrm{mg}$ 4,4'-Bis(2-benzoxazolyl)stilbene, $300 \mathrm{ng} / \mathrm{mg}$ for DIDS, $130 \mathrm{ng} / \mathrm{mg}$ for $E$ - $\alpha$-methylstilbene, and $35 \mathrm{ng} /$ $\mathrm{mg}$ for 2,4-dinitro-3',4'-(methylenedioxy)stilbene.

\section{Results}

3.1. Chloride channel transcripts identified in S. frugiperda, D. melanogaster, and $H$. sapiens

An analysis of chloride channel complexes through NCBI searches revealed a total of four, 39, and 68 transcripts annotated as chloride channel-encoding mRNAs for FAW, D. melanogaster, and H. sapiens, 


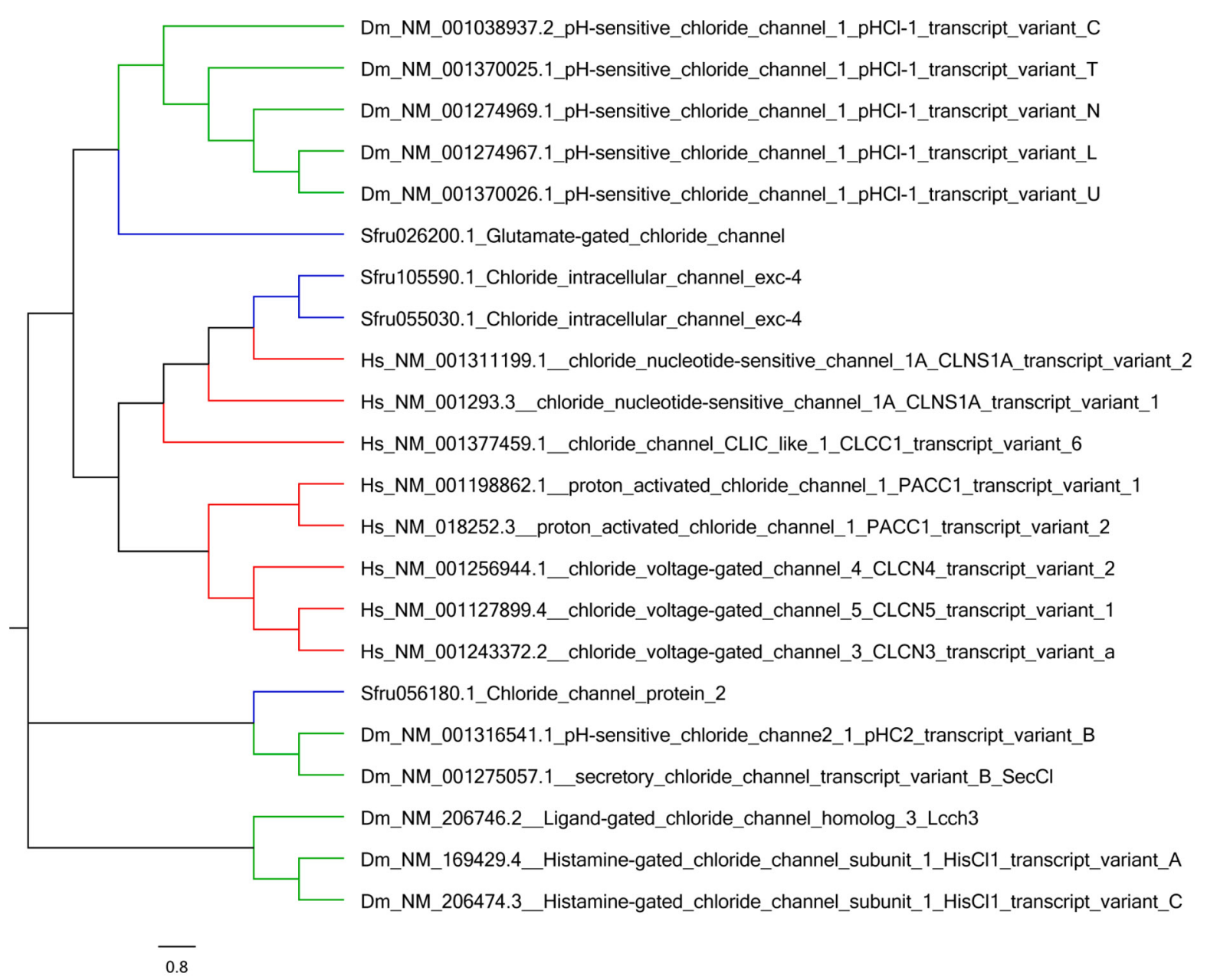

Figure 2. Neighbor-joining phylogenetic tree of select sequences annotated as chloride channels in Spodoptera frugiperda (blue), D. melanogaster (green), and H. sapiens (red).

respectively (Supplemental Table 1). A neighbor-joining phylogenetic tree of select chloride channel transcripts shows the alignment of four chloride channels from FAW orthologous to $10 \mathrm{D}$. melanogaster and eight $H$. sapiens chloride channels (Figure 2). The four chloride channels from FAW include one glutamate-gated chloride channel transcript orthologous to $\mathrm{pH}$-sensitive chloride channel transcripts of D. melanogaster, two intracellular chloride channel transcripts orthologous to nucleotide-sensitive, proton-activated, and voltage-gated chloride channel transcripts of $H$. sapiens, and one chloride channel 
transcript orthologous to $\mathrm{pH}$-sensitive, secretory, ligand-gated, and histamine-gated chloride channel transcripts of $D$. melanogaster (Figure 2).

\subsection{Potency of select chloride channel modulators to FAW central} neural activity

We aimed to establish baseline potency values for known modulators of LGCC and VDCC to spontaneous CNS firing of the LSU-Lab-1. The GABA-gated chloride channel complex is an exploited insecticide target and, thus, we compared the potency of GABA and fipronil as representative modulators of LGCC. As expected, GABA was shown to inhibit CNS firing at high micromolar to low millimolar concentrations with an $\mathrm{IC}_{50}$ of $0.94 \mathrm{mM}$ (95\% CI: 0.6-1.2 mM, Hillslope: -2.4 , $\left.r^{2}: 0.85\right)$ and the concentration-response curve and representative recording are shown in Figure 3A. Fipronil, a known blocker of GABAgated chloride channels, was highly potent against the FAW CNS firing rates with an $\mathrm{EC}_{50}$ of $149 \mathrm{nM}$ (95\% CI: 75-297 nM, Hillslope: 1.6, $r^{2}$ : 0.72). Maximal firing rates after exposure to fipronil was observed at $300 \mathrm{nM}$ and inhibition of nerve activity was observed at $1 \mu \mathrm{M}$ (Figure 3B). The organochlorine lindane was the most potent molecule studied to the FAW CNS activity with an $\mathrm{EC}_{50}$ of $6.6 \mathrm{nM}$ (95\% CI: 2.2-11.6 $\mathrm{nM}$, Hillslope: $0.83, r^{2}: 0.82$ ) with near maximal firing achieved at approximately $30 \mathrm{nM}$ (Figure $3 \mathrm{C}$ ).

\subsection{Potency of stilbenes to central neural activity of FAW}

The stilbene sulfonate DIDS (4,4'-diisothiocyanato-2,2'stilbenedisulfonic acid), which is an established VDCC chloride channel blocker (Cabantchik and Greger, 1992; Matulef and Maduke, 2005), was found to produce a biphasic response to CNS firing rates with lower concentrations yielding an increase in CNS firing rates and higher concentrations depressing CNS activity (Figure $3 \mathrm{~A}$ ). The $\mathrm{EC}_{50}$ of DIDS was found to be $41 \mu \mathrm{M}$ (95\% CI: 31-52 $\mu \mathrm{M}$, Hillslope: 3.9, $r^{2}$ : 0.74) whereas the concentration to inhibit $50 \%$ of CNS activity $\left(\mathrm{IC}_{50}\right.$ ) was $1.5 \mathrm{mM}$ (95\% CI: 0.6-3.6 mM, Hillslope: $-1.6, r^{2}$ : o.88). A representative recording showing DIDS-mediated excitation followed by depression of CNS firing rates is shown in Figure 4A. The potency of $E$ - and 

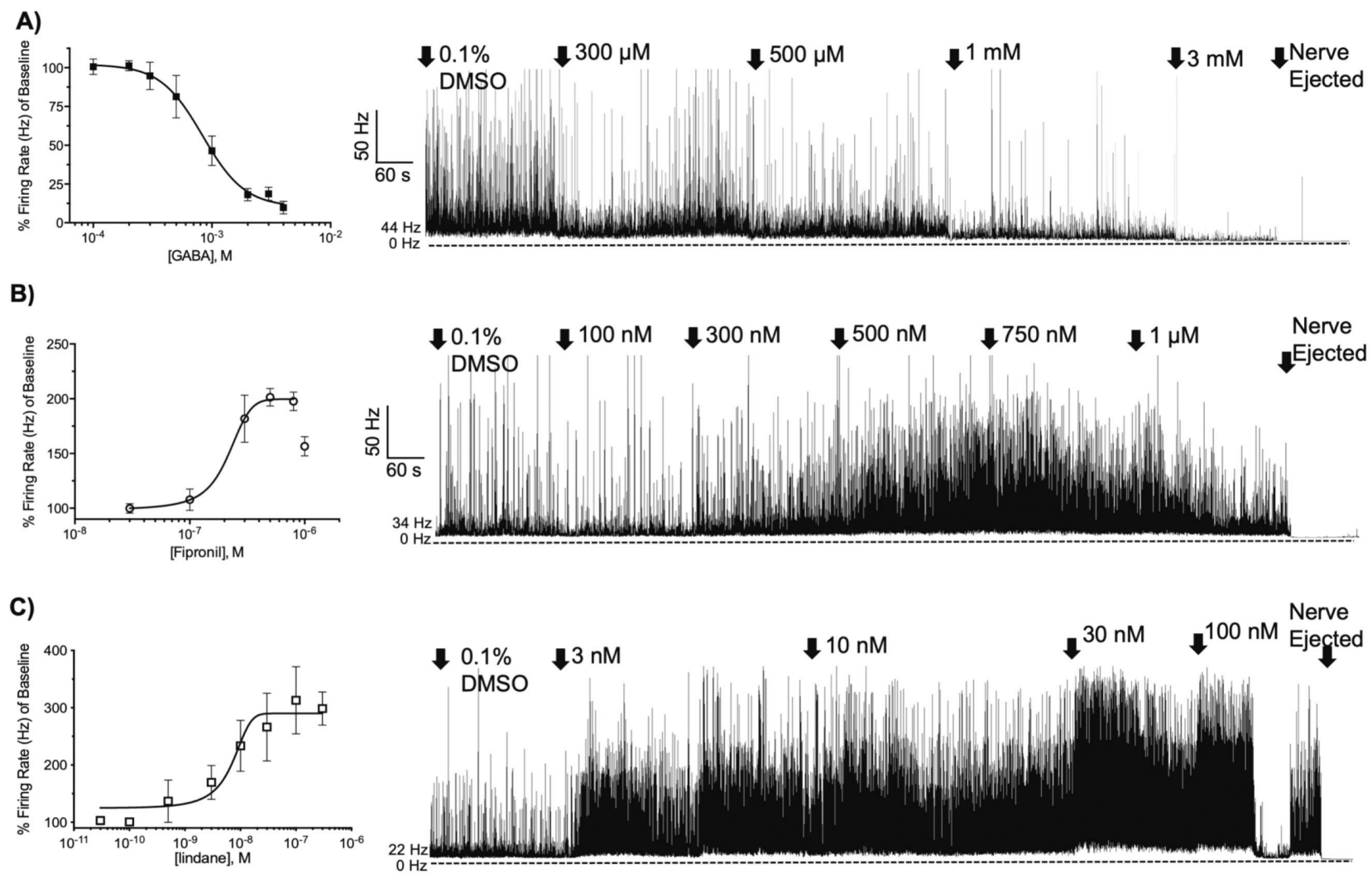

Figure 3. Potency determinations for select modulators of chloride channel complexes to FAW CNS firing rates. Concentration-response curves and representative recordings for GABA (A), fipronil (B), and lindane (C). Data points represent means from replicated recordings ( $n=5^{-12}$ preparations per curve, with each concentration replicated a minimum of 4 times). Data points represent mean percentage increase of baseline firing rate and error bars represent SEM.

$Z$ - stilbene to FAW central descending nerve activity was tested for comparison against the highly substituted stilbene, DIDS. Z-stilbene was found to be 4.3 -fold more potent when compared to DIDS with an $\mathrm{EC}_{50}$ value of $8.7 \mu \mathrm{M}$ (95\% CI: 6-13 $\mu \mathrm{M}$, Hillslope: 3.6, $r^{2}$ : 0.76). Maximal firing rate was observed at $30 \mu \mathrm{M}$ with a 2.2 -fold increase in firing over baseline activity (Figure 4B). Three of the seven recordings with $Z$-stilbene showed a biphasic response after exposure of the FAW CNS to $300 \mu \mathrm{M}$ whereas four of the seven recordings showed neuroexcitation that was sustained over the 3-5 min recording window. Of the three that showed a biphasic response, we observed an initial increase in firing frequency of approximately $20 \%$ when compared to 


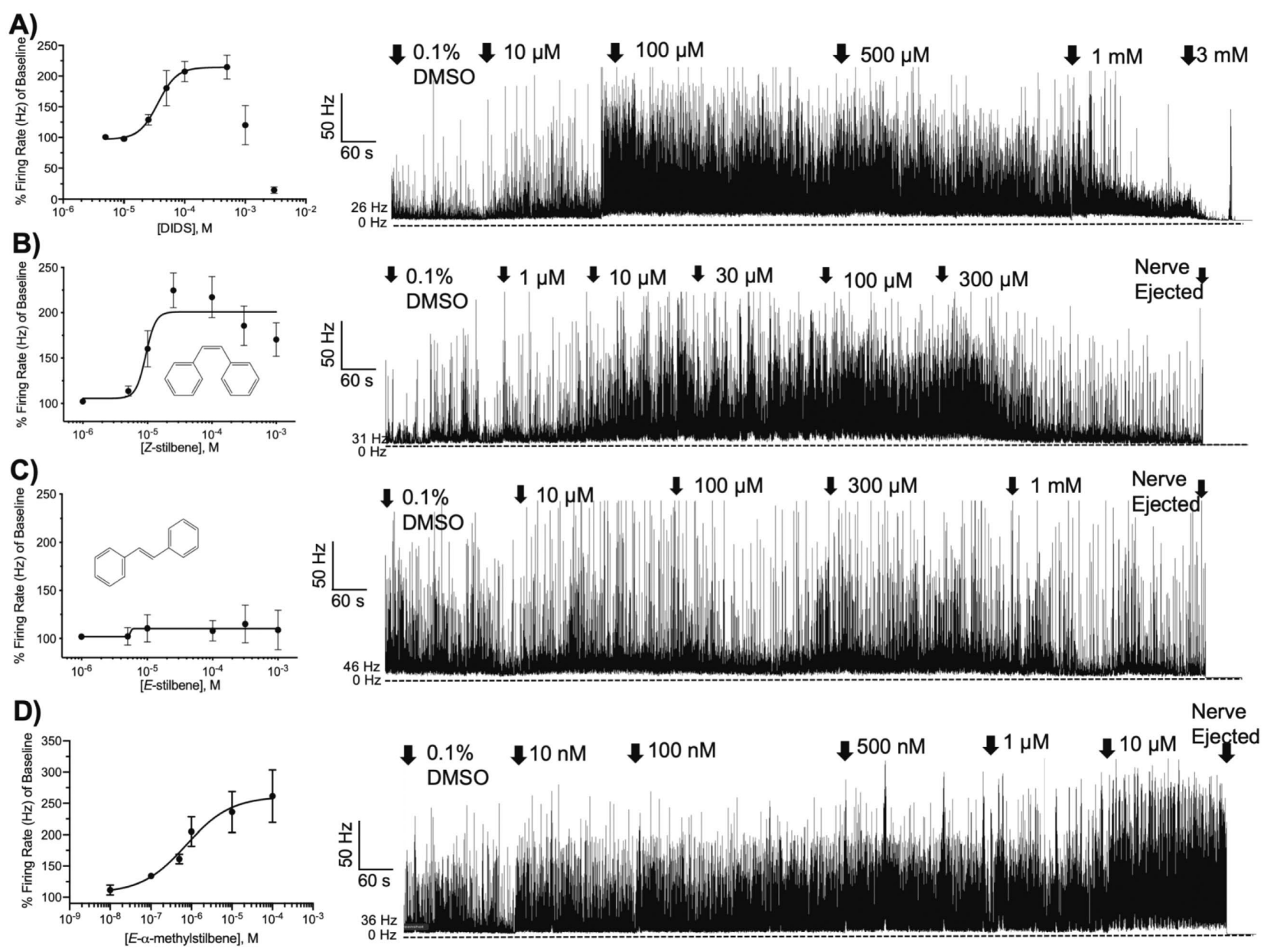

Figure 4. Potency determinations for stilbene molecules to FAW CNS firing rates. Concentration-response curves and representative recordings for DIDS (A), $Z$-stilbene (B), E-stilbene (C), and $E$ - $\alpha$-methylstilbene. The stilbene isomer structures are shown as an inset of panels B and C. Data points represent means from replicated recordings ( $n=5^{-12}$ preparations per curve, with each concentration replicated a minimum of 4 times). Data points represent mean percentage increase of baseline firing rate and error bars represent SEM. For DIDS, the inhibition of CNS firing at 1 and $3 \mathrm{mM}$ were excluded from the non-linear regression analysis to ensure accurate generation of an $\mathrm{EC}_{50}$ and the concentrations ranging from $5 \mu \mathrm{M}$ to $100 \mu \mathrm{M}$ were excluded to generate the $\mathrm{IC}_{50}$ value.

the firing rate after exposure to $100 \mu \mathrm{M}$ that was maintained for approximately $60 \mathrm{~s}$ followed by approximately $40 \%$ decrease in firing frequency (Figure 4B). Interestingly, $E$-stilbene was inactive on the FAW central neurons with only an $8 \%$ increase in firing frequency 
at $1 \mathrm{mM}$, which was the highest concentration tested (Figure $4 \mathrm{C}$ ). Although $E$-stilbene was inactive to the FAW, the slightly substituted analog $E$ - $\alpha$-methylstilbene was the most potent stilbene studied with an $\mathrm{EC}_{50}$ of $789 \mathrm{nM}$ (95\% CI: 488-1058 nM, Hillslope: $\left.0.72, r^{2}: 0.78\right)$.

\subsection{Toxicity of LGCC- and VDCC- directed insecticides to FAW}

Fipronil was found to be the most toxic of the compounds tested with a topical $\mathrm{LD}_{50}$ of $44 \mathrm{ng} / \mathrm{mg}$ of caterpillar (Hillslope: 1.1, $r^{2}: 0.91$ ). The FAW cuticle was a rather significant barrier to fipronil penetration as we observed a 10-fold increase in toxicity after microinjection $\left(\mathrm{LD}_{50}\right.$ : $4.1 \mathrm{ng} / \mathrm{mg}$ of caterpillar, Hillslope: $\left.1.2, r^{2}: 0.88\right)$. The organochlorine lindane was approximately 2-fold less toxic when compared to fipronil with an $\mathrm{LD}_{50}$ of $75 \mathrm{ng} / \mathrm{mg}$ of caterpillar (Hillslope: 1.3, $r^{2}$ : 0.92). Microinjection of lindane resulted in a 1.4-fold increase in toxicity, which was not statistically significant when compared to topical application. Topical application of any stilbenes tested did not result in appreciable toxicity at the highest tested dose (see methods for doses, Table 1). Microinjection marginally improved toxicity of stilbenes with the largest increase in toxicity being observed with $Z$-stilbene, which increased from $0 \%$ to $26 \pm 14 \%$ at $300 \mathrm{ng} / \mathrm{mg}$ of caterpillar (Table 1 ). Although we did not observe acute toxicity with stilbenes, hyperexcitation and uncoordinated movements were observed in caterpillars after topical application and microinjection treatment of $\alpha$-phenylcinnamonitrile, E- $\alpha$-methylstilbene, dintro2,4-dinitro-3',4'- (methylenedioxy)stilbene, and $Z$-stilbene.

\subsection{Synergized toxicity of stilbenes to FAW}

The lack of toxicity yet behavior indicating poisoning suggests metabolism of stilbenes may be preventing acute toxicity and, thus, the metabolic activity of cytochrome $\mathrm{P} 45 \mathrm{O}$ to stilbenes was studied through co-injection of $1 \mu \mathrm{g}$ of pipronyl butoxide (PBO). Significant synergism was observed with DIDS and $Z$-stilbene with synergized injected dose resulting in $50 \%$ lethality $\left(\mathrm{ID}_{50}\right.$ ) of $230 \mathrm{ng} / \mathrm{mg}$ of caterpillar (Hillslope: $1.8, r^{2}: 0.8$ ) and $340 \mathrm{ng} / \mathrm{mg}$ of caterpillar Hillslope: $1.5, r^{2}: 0.7$ ), respectively. Unfortunately, $\mathrm{ID}_{50}$ values were not able to be generated for other stilbenes due to less than 50\% mortality at the highest dose 
Table 1 Toxicity of standard chloride channel inhibitors and stilbene analogs to FAW after topical application and microinjection.

\begin{tabular}{|c|c|c|c|}
\hline \multirow[t]{2}{*}{ Compound } & $\begin{array}{l}\text { Topical } \\
\text { Toxicity }\end{array}$ & $\begin{array}{l}\text { Injected } \\
\text { Toxicity }\end{array}$ & $\begin{array}{l}+P B O^{3}(1 \mu \mathrm{g} / \\
\text { caterpillar })\end{array}$ \\
\hline & $\begin{array}{l}{ }^{1} \mathrm{LD}_{50}, \mathrm{ng} / \mathrm{mg} \\
\text { insect }(95 \% \mathrm{Cl})\end{array}$ & $\begin{array}{l}{ }^{2} D_{50}, \mathrm{ng} / \mathrm{mg} \\
\text { insect }(95 \% \mathrm{Cl})\end{array}$ & $\begin{array}{l}I D_{50}, \mathrm{ng} / \mathrm{mg} \\
\text { insect }(95 \% \mathrm{Cl})\end{array}$ \\
\hline Fipronil & $44(34-53)$ & $4.1(2.6-5.4)$ & - \\
\hline Lindane & $75(52-104)$ & $52(40-65)$ & - \\
\hline \pm DIDS & $0 \%$ & $13 \pm 2 \%$ & $230(140-320)$ \\
\hline$Z$-stilbene & $0 \%$ & $26 \pm 14 \%$ & $340(225-410)$ \\
\hline E-stilbene & $0 \%$ & $10 \pm 7 \%$ & $6 \pm 2 \%$ \\
\hline dintrostilbene & $0 \%$ & $6 \pm 3 \%$ & $45 \pm 7 \%$ \\
\hline$E$ - $\alpha$-methylstilbene & $0 \%$ & $6 \pm 5 \%$ & $48 \pm 12 \%$ \\
\hline$\alpha$-phenylcinnamonitrile & $0 \%$ & $17 \pm 4 \%$ & $25 \pm 6 \%$ \\
\hline Bis-stilbene & $0 \%$ & $3 \pm 1 \%$ & $20 \pm 7 \%$ \\
\hline
\end{tabular}

Full dose-response curves were generated where solubility of the molecule permitted and corresponding LD50/ID50 values are presented. If solubility prevented construction of a full curve, then mortality is presented in $\% \pm$ SD at solubility limits.

1. $L C_{50}$ : lethal concentration that elicits $50 \%$ mortality.

2. ID ${ }_{50}$ : Injected dose that elicits $50 \%$ mortality.

3. PBO: piperonyl butoxide.

studied, but an increased toxicity of 2- to 8-fold was observed for all remaining stilbenes except for $E$-stilbene (Table 1). Lastly, 2,4-dinitro$3^{\prime}, 4^{\prime}$-(methylenedioxy)stilbene that resulted in $45 \pm 7 \%$ toxicity after coinjection with $\mathrm{PBO}$ at $35 \mathrm{ng} / \mathrm{mg}$ of caterpillar, which is approximately 4 -fold more toxic than DIDS at the corresponding dose and is similar to the non-synergized toxicity of lindane (Table 1).

\subsection{Growth and developmental effects of stilbenes}

To measure the sub-lethal effect of stilbenes on FAW, we tested aspects of development such as the time of larval development and the functional capacity of emerged adults. We found 2,4-dinitro-3',4'(methylenedioxy)stilbene, 4,4'-bis(2-benzoxazolyl)stilbene, and $\alpha$-phenylcinnamonitrile to significantly $(P<0.05)$ reduce the larval development time by 1.4-, 1.6-, and 1.6-fold whereas no statistical difference in larval development time for $Z$ - and $E$-stilbene, $E$ - $\alpha$-methylstilbene, or DIDS (Figure $5 \mathrm{~A}$ ). FAW individuals reared on $E$ - $\alpha$-methylstilbene, 2,4-dinitro-3',4'-(methylenedioxy)stilbene, 
A)

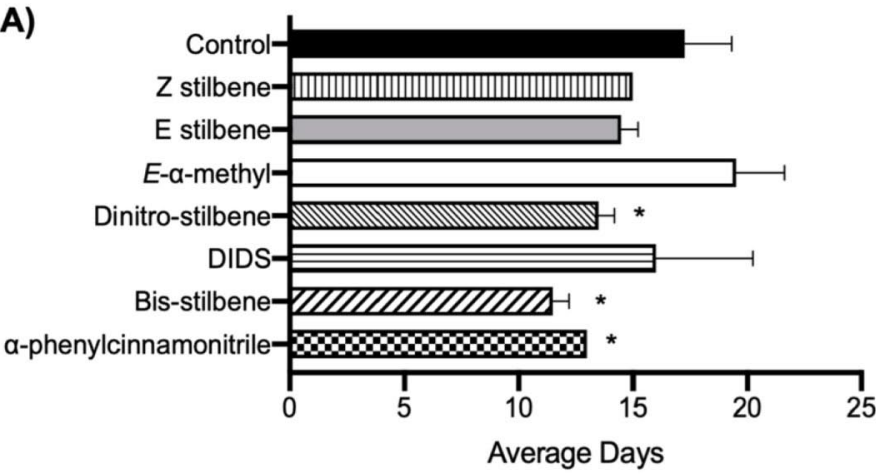

B)

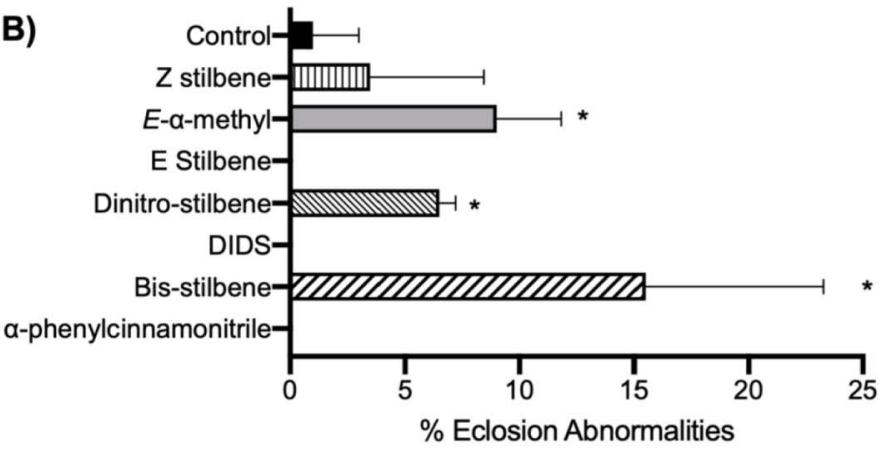

C)

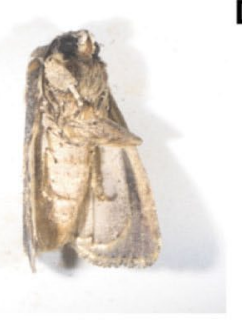

D)

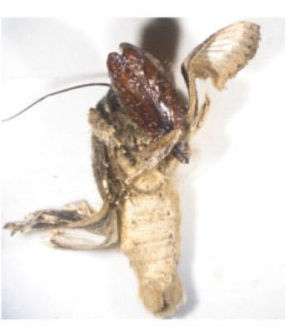

E)

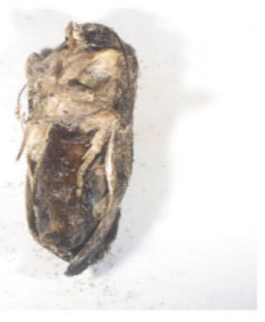

Figure 5. Developmental effects of stilbenes. A) FAW larval developmental time period from neonate to pupation. B) Developmental abnormalities after eclosion. Bars for panels A and B represent mean ( $n=30-50$ individuals) adults that attempted adult eclosion. Error bars in panels A-B represent standard error of mean. C-E) Representative images of adults affected by larval exposure to control, 2,4-dinitro-3',4'(methylenedioxy)stilbene, and 4,4'-Bis(2-benzoxazolyl) stilbene. All compounds were studied at $100 \mu \mathrm{g} / \mathrm{mL}$ of media.

bisstilbene were shown to have a significantly increased percentage of developmental abnormalities upon eclosion with $9 \%, 7 \%$, and $16 \%$ adults incapable of flight, respectively (Figure 5B). Control moths displayed abnormalities upon emergence at a rate of $2 \pm$ 
0.6\% Representative images for abnormalities of $E$ - $\alpha$-methylstilbene, 2,4-dinitro-3',4'-(methylenedioxy)stilbene, and 4,4'-bis(2-benzoxazolyl)stilbene are shown in Figure 5C, D, and E, respectively.

\section{Discussion}

The identification of new chemistries that augment the suite of products commercialized for FAW management is of significant interest considering the expansion of FAW across the world, the few available classes of chemistry, and the rapid development of resistance to these chemistries (Goergen et al., 2016; Sharanabasappa et al., 2018; Carvalho et al., 2013; I.I.R.A. Committee, 2016). The phthalic and anthranilic acid diamides, such as flubendiamide and chlorantraniliprole, were introduced in 2007 and quickly became widely used products due to established pyrethroid and organophosphate resistance. Although crossresistance from pyrethroids and organophosphates was not observed with diamides, ca. 500-fold resistance to diamides has been documented in multiple species of lepidopteran pests that has been attributed to control failure (Bolzan et al., 2019; Yao et al., 2017; Troczka et al., 2012; Wang and $\mathrm{Wu}, 2012$ ). The development of diamide resistance has continued to reduce the number of effective modes of action that are available to reduce FAW populations and underscores the need to identify new chemical leads that can be developed to reduce the economic burden of FAW. Chloride channels are a broad class of ion channels that are regulated by binding of ligands or membrane voltage that have presented multiple opportunities for agrochemical development, which is reviewed in (Bloomquist, 2003). While the LGCC have been heavily studied and are the targets of multiple insecticidal classes, the VDCC have only recently been considered promising candidates for insecticide development ( $\mathrm{Vu}$ et al., 2020; Boina and Bloomquist, 2009).

A neighbor-joining phylogenetic tree representing the multiple sequence alignment of genome-annotated chloride channel complexes revealed four ligand-gated and voltage-dependent chloride channel transcripts for FAW compared to the 39 and 68 chloride channel transcripts for Drosophila and humans (Figure 2, Supplemental Table 1). These LGCC and VDCC transcripts of FAW are orthologous to those 
of Drosophila and humans, which are likely to have similar functions and properties. However, comparative and functional genomics and pharmacological approaches are warranted to not only test these expectations, but to validate the structure-activity relationships and selectivity of new chemistries targeting chloride channel complexes.

In an effort to identify novel structural scaffolds that can be used to control FAW, we studied stilbene analogs, which are a class of naturally occurring polyphenols isolated from a variety of flowering plants resistant to insect attack (Singh et al., 2021). Interestingly, methanol extracts of the bark from Yucca periculosa yielded stilbene isolates that were shown to inhibit FAW growth and development as well as induce mortality (Torres et al., 2003; Lv et al., 2014). In line with these previous studies, select stilbenes were found to significantly reduce the time for larval development and significantly increase the emerged adults that displayed developmental abnormalities (Figure 5). These developmental impacts of select stilbenes may indicate activity at the ecdysteroid receptor (Lv et al., 2014; Dinan et al., 1999; Shen et al., 2009), yet more refined biochemical studies are needed to validate this correlation. Disulfonic stilbenes are established anion transporter and channel inhibitors that have been used to understand the physiology and toxicological relevance of mammalian (Cabantchik and Greger, 1992; Verkman and Galietta, 2009) and arthropod (Boina and Bloomquist, 2009) anion transporters. Thus, we employed $Z$ - and $E$ stilbene isomers as well as differently substituted stilbenes to identify scaffolds that can be used as a base molecule for development of VDCC-directed insecticides. A dramatic shift in potency to CNS firing between $Z$ - and $E$-stilbene isomers was observed (Figure 4 ) where $Z$ stilbene was more potent than DIDS, but $E$-stilbene had no appreciable effect to FAW CNS firing at concentrations up to $1 \mathrm{mM}$. Albeit low, the acute toxicity of stilbene isomers correlated with their respective CNS potencies with $Z$-stilbene inducing modest mortality at 300 $\mathrm{ng} / \mathrm{mg}$ of insect and $E$-stilbene inducing no mortality. Interestingly, $E$ - $\alpha$-methylstilbene, which has a single methyl substitution on the $E$ stilbene scaffold, induced neuroexcitation of central neurons at high nanomolar potency and induced relatively high toxicity after PBO synergism. These data suggest appropriate substitutions are more relevant to biological activity when compared to structural conformations. To further probe the utility of stilbene scaffolds as FAW toxicants, we 
studied the more substituted stilbene derivative DIDS against FAW CNS activity and survivorship. Extracellular recordings of central descending nerves showed DIDS induced excitation of FAW CNS activity at mid-micromolar concentrations, which may be due to an inability to maintain electrical potentials after inhibition of VDCC. However, the inhibition of CNS firing observed with higher concentrations of DIDS indicates ion secretion may be altered leading to intracellular acidification and decreased depolarization, which has been an observed action of DIDS against the leech CNS (Deitmer, 1991). Despite the moderate potency to FAW CNS firing rates, DIDS only induced appreciable toxicity after co-injection with PBO. This is somewhat surprising considering the paralytic activity observed in other lepidopteran species (Boina and Bloomquist, 2009) and acute toxicity to mites ( $\mathrm{Vu}$ et al., 2020). The metabolic product of DIDS, 4,4'-diaminostilbene2,2'-disulfonic acid (DADS), which is a compound with the isothiocyanate groups of DIDS hydrolyzed to primary amines, has no potency to mammalian chloride channel $(\mathrm{ClC})$ proteins and potentially explains the lack of FAW toxicity in the absence of PBO (Wulff, 2008; Matulef et al., 2008). However, it is interesting to note that the reaction between DADS and DIDS yields multimers of DIDS and the higher molecular weight pentamer is 120 -fold more potent than the DIDS monomer against mammalian ClC proteins (Wulff, 2008; Matulef et al., 2008). The tetrameric and pentameric derivatives of DIDS are not likely to be suitable for agrochemical use due to the high molecular weight and that expected charged state upon entering the hemolymph. However, these multimers may represent probes to study the biophysics and protein structure of arthropod chloride channels that can be used to guide design of arthropod potent and selective chloride channel targeting insecticides.

The data presented in this study indicate chloride channel complexes contribute to FAW CNS function and, thus, FAW control and insecticide resistance management programs would benefit from future work aiming to develop insecticides targeting chloride channels. While patch clamp electrophysiology studies remain to be performed to validate VGCC as targets of stilbenes, these data establish a platform for potential development of novel chemical probes to study FAW neural signaling by adding to the relatively small body of knowledge describing the potency of select chloride channel modulators to FAW neural 
activity. Lastly, although the modest toxicity of the stilbenes studied reduces enthusiasm for these scaffolds to be developed into FAW specific insecticides, the enhancement of toxicity after coinjection with PBO and comparatively high synergized toxicity of 2,4-dinitro-3', $4^{\prime}-$ (methylenedioxy)stilbene and $E$ - $\alpha$-methylstilbene (Table 1) provides justification for further investigation of stilbene chemistry to identify lead scaffolds for development.

Funding Funding provided by USDA Hatch grant number CT-0273 to D.R. Swale (project \# 94313).

Competing interests The authors declare no conflicts of interest.

Appendix A. Supplementary data to this article can be found following the References.

\section{References}

Abalis, I.M., Eldefrawi, A.T., Eldefrawi, M.E., 1986. Actions of avermectin B1a on the gamma-aminobutyric acidA receptor and chloride channels in rat brain. J. Biochem. Toxicol. 1, 69-82.

Abbot, W.S., 1925. A method of computing the effectiveness of an insecticide. J. Econ. Entomol. 18, 265-267.

Asahi, M., Kobayashi, M., Matsui, H., Nakahira, K., 2015. Differential mechanisms of action of the novel gamma-aminobutyric acid receptor antagonist ectoparasiticides fluralaner (A1443) and fipronil. Pest Manag. Sci. 71, 91-95.

Blanco, C.A., et al., 2016. Current situation of pests targeted by Bt crops in Latin America. Curr. Opin. Insect. Sci. 15, 131-138.

Bloomquist, J.R., 1993. Toxicology, mode of action and target site-mediated resistance to insecticides acting on chloride channels. In: Comparative Biochemistry and Physiology. C, Comparative Pharmacology and Toxicology, 106, pp. 301-314.

Bloomquist, J.R., 1996. Ion channels as targets for insecticides. Annu. Rev. Entomol. 41, 163-19o.

Bloomquist, J.R., 2003. Chloride channels as tools for developing selective insecticides. Arch. Insect Biochem. Physiol. 54, 145-156.

Boaventura, D., et al., 2020. Detection of a ryanodine receptor target-site mutation in diamide insecticide resistant fall armyworm, Spodoptera frugiperda. Pest Manag. Sci. 76, 47-54. 
Boina, D.R., Bloomquist, J.R., 2009. Toxicity and disruption of midgut physiology in caterpillar of the European corn borer, Ostrinia nubilalis, by anion transporter blockers. Arch. Insect Biochem. Physiol. 70, 151-161.

Bolzan, A., et al., 2019. Selection and characterization of the inheritance of resistance of Spodoptera frugiperda (Lepidoptera: Noctuidae) to chlorantraniliprole and crossresistance to other diamide insecticides. Pest Manag. Sci. 75, 2682-2689.

Brookes, G., Barfoot, P., 2016. Environmental impacts of genetically modified (GM) crop use 1996-2014: impacts on pesticide use and carbon emissions. GM Crops Food 7, 84-116.

Cabantchik, Z.I., Greger, R., 1992. Chemical probes for anion transporters of mammalian cell membranes. Am. J. Phys. 262, C803-C827.

Cairns, J.E., et al., 2013. Adapting maize production to climate change in subSaharan Africa. Food Secur. 5, 1-16.

Carvalho, R.A., Omoto, C., Field, L.M., Williamson, M.S., Bass, C., 2013. Investigating the molecular mechanisms of organophosphate and pyrethroid resistance in the fall armyworm Spodoptera frugiperda. PLoS One 8, e62268.

Casida, J.E., Durkin, K.A., 2015. Novel GABA receptor pesticide targets. Pestic. Biochem. Physiol. 121, 22-30.

Deitmer, J.W., 1991. Electrogenic sodium-dependent bicarbonate secretion by glial cells of the leech central nervous system. J. Gen. Physiol. 98, 637-655.

Dinan, L., Savchenko, T., Whiting, P., Sarker, S.D., 1999. Plant natural products as insect steroid receptor agonists and antagonists. Pestic. Sci. 55, 331-335.

Fahlke, C., 2001. Ion permeation and selectivity in ClC-type chloride channels. Am. J. Physiol. Ren. Physiol. 280, F748-F757.

Goergen, G., Kumar, P.L., Sankung, S.B., Togola, A., Tamo, M., 2016. First report of outbreaks of the fall armyworm Spodoptera frugiperda (J E smith) (Lepidoptera, Noctuidae), a new alien invasive Pest in west and Central Africa. PLoS One 11, e0165632.

Gordy, J.W., Leonard, B.R., Blouin, D., Davis, J.A., Stout, M.J., 2015. Comparative effectiveness of potential elicitors of plant resistance against Spodoptera frugiperda (J. E. Smith) (Lepidoptera: Noctuidae) in four crop plants. PLoS One 10 e0136689.

Gutierrez-Moreno, R., et al., 2019. Field-evolved resistance of the fall armyworm (Lepidoptera: Noctuidae) to synthetic insecticides in Puerto Rico and Mexico. J. Econ. Entomol. 112, 792-802.

Houngbo, S., et al., 2020. Farmers' knowledge, perceptions, and management practices of the new invasive pest, fall armyworm (Spodoptera frugiperda) in Ethiopia and Kenya. Agriculture 10, 430-445.

Hu, K., Li, J., Webster, J., 1999. Nematicidal metabolites produced by Photorhabdus luminescens (Enterobacteriaceae), bacterial symbiont of entomopathogenic nematodes. Nematology 1, 457-469.

I.I.R.A. Committee. (2016). https://irac-online.org/ 
Jia, B., et al., 2009. Inheritance, fitness cost and mechanism of resistance to tebufenozide in Spodoptera exigua (Hubner) (Lepidoptera: Noctuidae). Pest Manag. Sci. 65, 996-1002.

Jiang, S., Tsikolia, M., Bernier, U.R., Bloomquist, J.R., 2017. Mosquitocidal activity and mode of action of the Isoxazoline Fluralaner. Int. J. Environ. Res. Public Health 14 .

Kansiime, M.K., et al., 2019. Farmer perception of fall armyworm (Spodoptera frugiperda J.E. Smith) and farm-level management practices in Zambia. Pest Manag. Sci. 75, 2840-2850.

Lv, X.-Q., Feng, G., Liu, Y.-Q., Nan, X., Yang, L., 2014. CA-4, a natural cis-stilbene compound with potential insecticidal activity. Med. Chem. Res. 23, 3347-3352.

Matulef, K., Maduke, M., 2005. Side-dependent inhibition of a prokaryotic $\mathrm{ClC}$ by DIDS. Biophys. J. 89, 1721-1730.

Matulef, K., et al., 2008. Discovery of potent CLC chloride channel inhibitors. ACS Chem. Biol. 3, 419-428.

McComic, S.E., Rault, L.C., Anderson, T.D., Swale, D.R., 2020. Reduced neuronal sensitivity and susceptibility of the fall armyworm, Spodoptera frugiperda, to pyrethroids in the absence of known knockdown mutations. Pestic. Biochem. Physiol. 169, 104652.

Miglianico, M., et al., 2018. Repurposing isoxazoline veterinary drugs for control of vector-borne human diseases. Proc. Natl. Acad. Sci. U. S. A. 115, E6920-E6926.

Nakao, T., Banba, S., 2016. Broflanilide: a meta-diamide insecticide with a novel mode of action. Bioorg. Med. Chem. 24, 372-377.

Nakao, T., Banba, S., Nomura, M., Hirase, K., 2013. Meta-diamide insecticides acting on distinct sites of RDL GABA receptor from those for conventional noncompetitive antagonists. Insect Biochem. Mol. Biol. 43, 366-375.

Okuma, D.M., et al., 2018. Inheritance and fitness costs of Spodoptera frugiperda (Lepidoptera: Noctuidae) resistance to spinosad in Brazil. Pest Manag. Sci. 74, 1441-1448.

Ozoe, Y., Asahi, M., Ozoe, F., Nakahira, K., Mita, T., 2010. The antiparasitic isoxazoline A1443 is a potent blocker of insect ligand-gated chloride channels. Biochem. Biophys. Res. Commun. 391, 744-749.

Ozoe, Y., et al., 2013. Insecticidal 3-benzamido- $\mathrm{N}$-phenylbenzamides specifically bind with high affinity to a novel allosteric site in housefly GABA receptors. Pestic. Biochem. Physiol. 107, 285-292.

Rios-Diez, J.D., Saldamando-Benjumea, C.I., 2011. Susceptibility of Spodoptera frugiperda (Lepidoptera: Noctuidae) strains from Central Colombia to two insecticides, methomyl and lambda-cyhalothrin: a study of the genetic basis of resistance. J. Econ. Entomol. 104, 1698-1705.

Salgado, V.L., Sheets, J.J., Watson, G.B., Schmidt, A.L., 1998. Studies on the mode of action of Spinosad: the internal effective concentration and the concentration dependence of neural excitation. Pestic. Biochem. Physiol. 6o, 103-110. 
Santos-Amaya, O.F., et al., 2017. Magnitude and allele frequency of Cry1F resistance in Field populations of the fall armyworm (Lepidoptera: Noctuidae) in Brazil. J. Econ. Entomol. 110, 1770-1778.

Sharanabasappa, et al., 2018. First report of the fall armyworm, Spodoptera frugiperda (JE Smith) (Lepidoptera: Noctuidae), an alien invasive pest on maize in India. Pest. Manag. Hortic. Ecosyst. 24, 23-29.

Shen, T., Wang, X.N., Lou, H.X., 2009. Natural stilbenes: an overview. Nat. Prod. Rep. 26, 916-935.

Sievers, F., et al., 2011. Fast, scalable generation of high-quality protein multiple sequence alignments using Clustal omega. Mol. Syst. Biol. 7, 539.

Singh, S., Kaur, I., Kariyat, R., 2021. The multifunctional roles of polyphenols in plantherbivore interactions. Int. J. Mol. Sci. 22, 1442.

Torres, P., et al., 2003. Antioxidant and insect growth regulatory activities of stilbenes and extracts from Yucca periculosa. Phytochemistry 64, 463-473.

Troczka, B., et al., 2012. Resistance to diamide insecticides in diamondback moth, Plutella xylostella (Lepidoptera: Plutellidae) is associated with a mutation in the membrane-spanning domain of the ryanodine receptor. Insect Biochem. Mol. Biol. 42, 873-880.

Verkman, A.S., Galietta, L.J., 2009. Chloride channels as drug targets. Nat. Rev. Drug Discov. 8, 153-171.

Vu, P.D., Rault, L.C., Jenson, L.J., Bloomquist, J.R., Anderson, T.D., 2020. Voltagegated chloride channel blocker DIDS as an acaricide for Varroa mites. Pestic. Biochem. Physiol. 167, 104603.

Wang, X., Wu, Y., 2012. High levels of resistance to chlorantraniliprole evolved in field populations of Plutella xylostella. J. Econ. Entomol. 105, 1019-1023.

$\mathrm{Wu}, \mathrm{Q} . \mathrm{L}$. , et al., 2019. Estimation of the potential infestation area of newlyinvaded fall armyworm Spodoptera frugiperda in the Yangtze River valley of China. Insects 10.

Wulff, H., 2008. New light on the "old" chloride channel blocker DIDS. ACS Chem. Biol. 3, 399-401.

Xiao, H., et al., 2020. The genetic adaptations of fall armyworm Spodoptera frugiperda facilitated its rapid global dispersal and invasion. Mol. Ecol. Resour. 20, 1050-1068.

Yao, R., et al., 2017. Monitoring and mechanisms of insecticide resistance in Chilo suppressalis (Lepidoptera: Crambidae), with special reference to diamides. Pest Manag. Sci. 73, 1169-1178.

Yu, S.J., Nguyen, S.N., Abo-Elghar, E.G., 2003. Biochemical characteristics of insecticide resistance in the fall armyworm, Spodoptera frugiperda. Pestic. Biochem. Physiol. 77, 1-11. 


\section{Supplemental Tables}

Chloride channels as targets to control the fall armyworm, Spodoptera frugiperda

Authors: Sarah E. McComic ${ }^{1}$, Leslie C. Rault ${ }^{2}$, Troy D. Anderson ${ }^{2}$, Daniel R. Swale ${ }^{1 *}$

\section{Author Affiliations:}

${ }^{1}$ Louisiana State University AgCenter, Department of Entomology, Baton Rouge, LA 70803

${ }^{2}$ University of Nebraska, Department of Entomology, 103 Entomology Hall, Lincoln, NE 68583 
Supplementary Table 1: Full list of the transcripts annotated as chloride channels in the transcriptomes of Spodoptera frugiperda, Drosophila melanogaster, and Homo sapiens. Their NCBI accession numbers are indicated.

\begin{tabular}{|c|c|}
\hline Organism & Organism abbreviation, Accession number and annotation \\
\hline $\begin{array}{l}\text { Spodoptera } \\
\text { frugiperda }\end{array}$ & $\begin{array}{l}\text { Sfru026200.1_Glutamate-gated_chloride_channel } \\
\text { Sfru105590.1_Chloride_intracellular_channel_exc-4 } \\
\text { Sfru055030.1_Chloride_intracellular_channel_exc-4 } \\
\text { Sfru056180.1_Chloride_channel_protein_2 }\end{array}$ \\
\hline $\begin{array}{l}\text { Drosophila } \\
\text { melanogaster }\end{array}$ & $\begin{array}{l}\text { Dm_NM_001038934.4_pH-sensitive_chloride_channel_1_pHCl-1_transcript_variant_D } \\
\text { Dm_NM_001038935.3_pH-sensitive_chloride_channel_1_pHCl-1_transcript_variant_F } \\
\text { Dm_NM_001038936.4_pH-sensitive_chloride_channel_1_pHCl-1_transcript_variant_E } \\
\text { Dm_NM_001038937.2_pH-sensitive_chloride_channel_1_pHCl-1_transcript_variant_C } \\
\text { Dm_NM_001104281.2_chloride_channel-a_transcript_variant_G_ClC-a } \\
\text { Dm_NM_001104282.3_chloride_channel-a_transcript_variant_F_ClC-a } \\
\text { Dm_NM_001169979.1_pH-sensitive_chloride_channel_1_pHCl-1_transcript_variant_G } \\
\text { Dm_NM_001170120.2_Histamine } \\
\text { gated_chloride_channel_subunit_1_HisCl1_transcript_variant_D } \\
\text { Dm_NM_001170121.1_chloride_channel-a_transcript_variant_H_ClC-a } \\
\text { Dm_NM_001259852.1_pH-sensitive_chloride_channel_1_pHCl-1_transcript_variant_H } \\
\text { Dm_NM_001259853.1_pH-sensitive_chloride_channel_1_pHCl-1_transcript_variant_I } \\
\text { Dm_NM_001272608.2_chloride_intracellular_channel_transcript_variant_cClic } \\
\text { Dm_NM_001274965.1_pH-sensitive_chloride_channel_1_pHCl-1_transcript_variant_J } \\
\text { Dm_NM_001274966.1_pH-sensitive_chloride_channel_1_pHCl-1_transcript_variant_K } \\
\text { Dm_NM_001274967.1_pH-sensitive_chloride_channel_1_pHCl-1_transcript_variant_L } \\
\text { Dm_NM_001274968.1_pH-sensitive_chloride_channel_1_pHCl-1_transcript_variant_M } \\
\text { Dm_NM_001274969.1_pH-sensitive_chloride_channel_1_pHCl-1_transcript_variant_N } \\
\text { Dm_NM_001274970.1_pH-sensitive_chloride_channel_1_pHCl-1_transcript_variant_O } \\
\text { Dm_NM_001274971.1_pH-sensitive_chloride_channel_1_pHCl-1_transcript_variant_P } \\
\text { Dm_NM_001274993.1__chloride_channel-c_transcript_variant_dClC-c } \\
\text { Dm_NM_001275057.1_secretory_chloride_channel_transcript_variant_bSecCl } \\
\text { Dm_NM_001275558.1__chloride_channel-a_transcript_variant_I_ClC-a } \\
\text { Dm_NM_001316541.1_pH-sensitive_chloride_channe2_1_pHC2_transcript_variant_B } \\
\text { Dm_NM_001370024.1_pH-sensitive_chloride_channel_1_pHCl-1_transcript_variant_S } \\
\text { Dm_NM_001370025.1_pH-sensitive_chloride_channel_1_pHCl-1_transcript_variant_T } \\
\text { Dm_NM_001370026.1_pH-sensitive_chloride_channel_1_pHCl-1_transcript_variant_U } \\
\text { Dm_NM_132700.4_chloride_intracellular_channel_transcript_variant_aClic } \\
\text { Dm_NM_136954.4__chloride_channel-b_ClC-b } \\
\text { Dm_NM_140577.2_chloride_channel-c_transcript_variant_bClC-c } \\
\text { Dm NM 140727 2 } 5 \text { secretorv chloride channel transcrint variant aSecCl }\end{array}$ \\
\hline
\end{tabular}


Dm_NM_141859.4__Histaminegated_chloride_channel_subunit_1_HisCl1_transcript_variant_b

Dm_NM_143604.3_pH-sensitive_chloride_channel_2_pHCl-2_transcript_variant_A

Dm_NM_168650.2_chloride_channel-c_transcript_variant_cClC-c

Dm_NM_169429.4_Histamine-

gated_chloride_channel_subunit_1_HisCl1_transcript_variant_a

Dm_NM_169431.3_chloride_channel-a_transcript_variant_cClC-a

Dm_NM_169432.3_chloride_channel-a_transcript_variant_aClC-a

Dm_NM_176462.2_chloride_channel-a_transcript_variant_dClC-a

Dm_NM_206474.3_Histamine-

gated_chloride_channel_subunit_1_HisCl1_transcript_variant_c

Dm_NM_206746.2_Ligand-gated_chloride_channel_homolog_3_Lcch3

Hs_NM_000083.3_chloride_voltage-gated_channel_1_CLCN1_transcript_variant_1

Hs_NM_000084.5_chloride_voltage-gated_channel_5_CLCN5_transcript_variant_3

Hs_NM_000085.5_chloride_voltage-gated_channel_Kb_CLCNKB_transcript_variant_1

Hs_NM_001042704.2_chloride_voltage-

gated_channel_Ka_CLCNKA_transcript_variant_2

Hs_NM_001048210.3_chloride_channel_CLIC_like_1_CLCC1_transcript_variant_1

Hs_NM_001114086.2_chloride_intracellular_channel_5_CLIC5_transcript_variant_1

Hs_NM_001114331.2_chloride_voltage-gated_channel_7_CLCN7_transcript_variant_2

Hs_NM_001127898.4_chloride_voltage-gated_channel_5_CLCN5_transcript_variant_2

Hs_NM_001127899.4_chloride_voltage-gated_channel_5_CLCN5_transcript_variant_1

Hs_NM_001165945.2_chloride_voltage-

gated_channel_Kb_CLCNKB_transcript_variant_2

Hs_NM_001171087.3_chloride_voltage-gated_channel_2_CLCN2_transcript_variant_2

Hs_NM_001171088.3_chloride_voltage-gated_channel_2_CLCN2_transcript_variant_3

Hs_NM_001171089.3_chloride_voltage-gated_channel_2_CLCN2_transcript_variant_4

Hs_NM_001198862.1_proton_activated_chloride_channel_1_PACC1_transcript_variant_1

Hs_NM_001243372.2_chloride_voltage-gated_channel_3_CLCN3_transcript_variant_a

Hs_NM_001243374.1_chloride_voltage-gated_channel_3_CLCN3_transcript_variant_c

Hs_NM_001256023.1_chloride_intracellular_channel_5_CLIC5_transcript_variant_3

Hs_NM_001256944.1_chloride_voltage-gated_channel_4_CLCN4_transcript_variant_2

Hs_NM_001256959.2_chloride_voltage-gated_channel_6_CLCN6_transcript_variant_2

Hs_NM_001257139.2_chloride_voltage-

gated_channel_Ka_CLCNKA_transcript_variant_3

Hs_NM_001272102.2_chloride_voltage-gated_channel_5_CLCN5_transcript_variant_5

Hs_NM_001278202.2_chloride_channel_CLIC_like_1_CLCC1_transcript_variant_3

Hs_NM_001278203.1_chloride_channel_CLIC_like_1_CLCC1_transcript_variant_4

Hs_NM_001282163.1_chloride_voltage-gated_channel_5_CLCN5_transcript_variant_4

Hs_NM_001285.4_chloride_channel_accessory_1_CLCA1

Hs_NM_001286.5_chloride_voltage-gated_channel_6_CLCN6_transcript_variant_1

Hs_NM_001287.6_chloride_voltage-gated_channel_7_CLCN7_transcript_variant_1 
Hs_NM_001287593.1_chloride_intracellular_channel_1_CLIC1_transcript_variant_1 Hs_NM_001287594.1_chloride_intracellular_channel_1_CLIC1_transcript_variant_3 Hs_NM_001288.4_chloride_intracellular_channel_1_CLIC1_transcript_variant_2 Hs_NM_001289.6_chloride_intracellular_channel_2_CLIC2 Hs_NM_001293.3_chloride_nucleotidesensitive_channel_1A_CLNS1A_transcript_variant_1

Hs_NM_001311199.1_chloride_nucleotidesensitive_channel_1A_CLNS1A_transcript_variant_2 Hs_NM_001311200.2_chloride_nucleotidesensitive_channel_1A_CLNS1A_transcript_variant_3

Hs_NM_001311201.2_chloride_nucleotidesensitive_channel_1A_CLNS1A_transcript_variant_4

Hs_NM_001311202.2_chloride_nucleotidesensitive_channel_1A_CLNS1A_transcript_variant_5

Hs_NM_001317009.2_chloride_intracellular_channel_6_CLIC6_transcript_variant_1 Hs_NM_001370649.1_chloride_intracellular_channel_5_CLIC5_transcript_variant_7 Hs_NM_001370650.1_chloride_intracellular_channel_5_CLIC5_transcript_variant_8 Hs_NM_001377458.1_chloride_channel_CLIC_like_1_CLCC1_transcript_variant_5 Hs_NM_001377459.1_chloride_channel_CLIC_like_1_CLCC1_transcript_variant_6 Hs_NM_001377460.1_chloride_channel_CLIC_like_1_CLCC1_transcript_variant_7 Hs_NM_001377461.1_chloride_channel_CLIC_like_1_CLCC1_transcript_variant_8 Hs_NM_001377462.1_chloride_channel_CLIC_like_1_CLCC1_transcript_variant_9 Hs_NM_001377463.1_chloride_channel_CLIC_like_1_CLCC1_transcript_variant_10 Hs_NM_001377464.1_chloride_channel_CLIC_like_1_CLCC1_transcript_variant_11 Hs_NM_001377465.1_chloride_channel_CLIC_like_1_CLCC1_transcript_variant_12 Hs_NM_001377466.1_chloride_channel_CLIC_like_1_CLCC1_transcript_variant_13 Hs_NM_001377467.1_chloride_channel_CLIC_like_1_CLCC1_transcript_variant_14 Hs_NM_001377468.1_chloride_channel_CLIC_like_1_CLCC1_transcript_variant_15 Hs_NM_001377469.1_chloride_channel_CLIC_like_1_CLCC1_transcript_variant_16 Hs_NM_001377470.1_chloride_channel_CLIC_like_1_CLCC1_transcript_variant_17 Hs_NM_001377478.1_proton_activated_chloride_channel_1_PACC1_transcript_variant_3 Hs_NM_001377479.1_proton_activated_chloride_channel_1_PACC1_transcript_variant_4 Hs_NM_001377480.1_proton_activated_chloride_channel_1_PACC1_transcript_variant_5 Hs_NM_001829.4_chloride_voltage-gated_channel_3_CLCN3_transcript_variant_b Hs_NM_001830.4_chloride_voltage-gated_channel_4_CLCN4_transcript_variant_1 Hs_NM_004070.4_chloride_voltage-gated_channel_Ka_CLCNKA_transcript_variant_1 Hs_NM_004366.6_chloride_voltage-gated_channel_2_CLCN2_transcript_variant_1 Hs_NM_004669.3_chloride_intracellular_channel_3_CLIC3 Hs_NM_006536.7_chloride_channel_accessory_2_CLCA2 Hs_NM_012128.4_chloride_channel_accessory_4_CLCA4_transcript_variant_1 Hs_NM_013943.3_chloride_intracellular_channel_4_CLIC4 Hs_NM_015127.5_chloride_channel_CLIC_like_1_CLCC1_transcript_variant_2 
Hs_NM_016929.5_chloride_intracellular_channel_5_CLIC5_transcript_variant_2 Hs_NM_018252.3_proton_activated_chloride_channel_1_PACC1_transcript_variant_2 Hs_NM_053277.3_chloride_intracellular_channel_6_CLIC6_transcript_variant_2 Hs_NM_173872.4_chloride_voltage-gated_channel_3_CLCN3_transcript_variant_e 